\title{
In situ control of electron gas dimensionality in freely suspended semiconductor membranes
}

\author{
Eva M. Höhberger ${ }^{\mathrm{a})}$ and Tomas Krämer \\ Center for NanoScience and Sektion Physik, Ludwig-Maximilians-Universität München, D-80539 München, \\ Germany \\ Werner Wegscheider \\ Institut für Angewandte und Experimentelle Physik, Universität Regensburg, D-93040 Regensburg, Germany \\ Robert H. Blick \\ Department of Electrical and Computer Engineering, University of Wisconsin-Madison, Madison, \\ Wisconsin 53706
}

(Received 4 March 2003; accepted 11 April 2003)

\begin{abstract}
We present fabrication and measurements of gated suspended low-dimensional electron systems. The core component of the device is a low-dimensional electron gas embedded in a free-standing beam processed from a GaAs/AlGaAs heterostructure. The dimensionality of the electronic system is fully controlled by a number of gating electrodes on the suspended membranes. Operation in the quantum Hall regime, in the one-dimensional case, and as zero-dimensional quantum dots is demonstrated. The resulting devices can be applied as ultrasensitive bolometers and as nanoelectromechanical circuits that reach the ultimate limits of displacement detection. (C) 2003 American Institute of Physics. [DOI: 10.1063/1.1580641]
\end{abstract}

Successive dimensional reduction of electron systems in semiconductor heterointerfaces led from the realization of high mobility transistors to the discovery of the quantum Hall effect, quantum point contacts (QPCs), and finally to quantum dots that operate as single electron transistors. ${ }^{1}$ In a similar way, the mechanical properties of semiconductor nanostructures can be distinctly modified by constraining the size of the crystalline host in one or more dimensions: In contrast to bulk material the phonon spectrum in these "phonon cavities" is split into discrete subbands and quantization effects become important for the thermal conductivitiy. ${ }^{2}$ Along with ongoing progress in nanoprocessing technology it has recently become possible to fabricate free-standing nanostructures with thickness of the order of only $100 \mathrm{~nm}$ in which these quantization effects have been observed. ${ }^{3-5}$

Gaining control of the phonon density of states opens up the possibility of designing a spectrum of lattice vibrations and, in particular, of tailoring the electron-phonon interaction in the phonon cavity. While certain phonon modes can be completely decoupled from the electronic system, the spontaneous emission of other modes can be strongly enhanced. ${ }^{6}$ Thus, phonon confinement seems to be a promising way by which to increase control of dissipation and dephasing processes in electronic systems which is an important, yet unsolved, problem for the realization of quantum

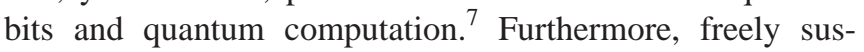
pended integrated devices that contain a low-dimensional electron system can be employed to obtain ultrasensitive bolometers ${ }^{4}$ and displacement detection close to the quantum limit. ${ }^{8-10}$

However, a combination of electron and phonon systems of reduced dimensionality has only been put forth recently.

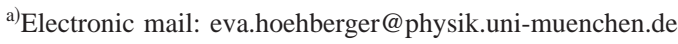

Since the fabrication of the earliest free-standing cantilevers that contained relatively large two-dimensional electron systems (2DEGs), ${ }^{11,12}$ mechanical properties of a suspended 2DEG resonator have been probed for smaller beams. ${ }^{13}$ Hallbars, ${ }^{14}$ ballistic cavities ${ }^{15}$ and quantum point contacts 9 have been integrated into suspended heterostructures. Recently, we reported on single electron tunneling in a freely suspended quantum dot that formed an electron-phonon cavity. ${ }^{8,16}$

Here we want to address fabrication and control of fully tunable gated low-dimensional electron systems in freely suspended GaAs/AlGaAs membranes. In these structures the dimension of the electron system can be continuously reduced to form a 2DEG, a quantum point contact, as well as single or double quantum dots thus allowing detailed investigation of dissipation in phonon cavities.

The starting material is a molecular beam epitaxially grown GaAs/AlGaAs heterostructure which contains two functional layer stacks, described elsewhere. ${ }^{8}$ The top part of the structure is the $130 \mathrm{~nm}$ thick active layer which will remain free standing after processing. It contains a deltadoped $\mathrm{GaAs} / \mathrm{Al}_{0.3} \mathrm{Ga}_{0.7} \mathrm{As}$ quantum well located $40 \mathrm{~nm}$ below the sample surface which sustains the 2DEG with an unilluminated sheet density of $n_{s}=9.1 \times 10^{11} \mathrm{~cm}^{-2}$ and mobility $\mu=234000 \mathrm{~cm}^{2} / \mathrm{V} \mathrm{s}$. Below, the sacrificial layer consisting of $400 \mathrm{~nm}$ of $\mathrm{Al}_{0.8} \mathrm{Ga}_{0.2} \mathrm{As}$ separates the active layer from the buffer.

Fabrication of the samples involves a series of processing steps starting with the application of AuGeNi ohmic contacts as well as thick Au bond pads by optical lithography. Subsequently, gate electrodes are defined along with highresolution position marks by electron beam lithography and covered with $50 \mathrm{~nm}$ of $\mathrm{Au}$. In the second step of electron beam lithography which is carefully aligned with the existing gate structures a mesa consisting of a beam with length $l$ 


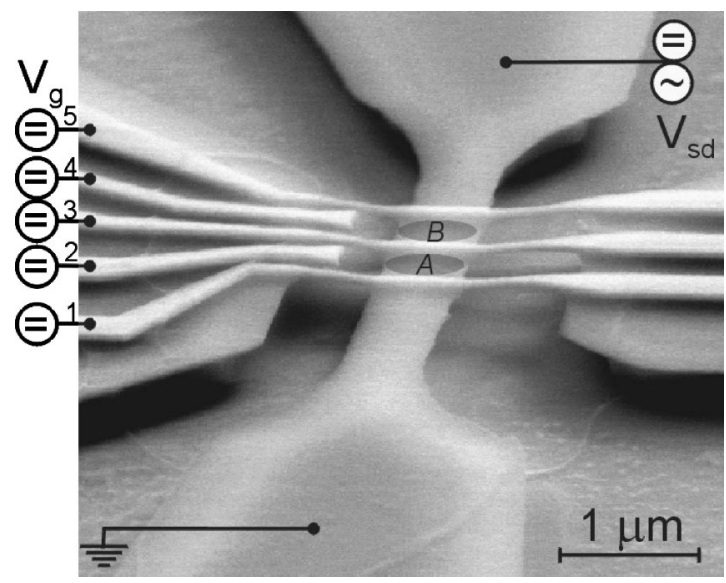

FIG. 1. Scanning electron micrograph of a freely suspended nanostructure that sustains a fully tunable low-dimensional electron system. A 2DEG is embedded in the $950 \mathrm{~nm}$ wide, $4 \mu \mathrm{m}$ long, $130 \mathrm{~nm}$ thick free-standing beam. Depletion of five equally suspended Au gate electrodes can be used to reduce the electronic dimension continuously from $2 \mathrm{D}, 1 \mathrm{D}$ to $0 \mathrm{D}$, allowing one to operate the device as 2DEG, QPC, single or double quantum dot.

$=4 \mu \mathrm{m}$ and width $w=950 \mathrm{~nm}$ is patterned and protected by a $60 \mathrm{~nm}$ thick $\mathrm{Ni}$ etch mask. Highly anisotripic inductive coupled plasma (ICP) reactive ion etching (RIE) with $\mathrm{SiCl}_{4}$ was employed to transfer the sample geometry into the 2DEG and through the sacrificial layer. In order to minimize edge depletion of the 2DEG and implantation of defects that lead to reduced electron mobility a radio frequency power of $10 \mathrm{~W}$ was used to keep the etching bias below $40 \mathrm{~V}$ whereas the plasma was generated at an ICP power of $30 \mathrm{~W}$. After dissolving the etch mask in a solution of $\mathrm{Fe}-(\mathrm{III})-\mathrm{Cl}$ the sacrificial layer is removed beneath the mesa and gate electrodes by wet etching in $0.1 \%$ hydrofluoric acid. The resulting free-standing structures are critical point dried in order to prevent rupture due to surface tension of the evaporating solvent.

A typical sample is displayed in Fig. 1. The scanning electron microscopy micrograph was taken at a steep angle to emphasize the three-dimensional character of the device. The $950 \mathrm{~nm}$ wide, $4 \mu \mathrm{m}$ long, $130 \mathrm{~nm}$ thick free-standing beam is clearly visible in the center of the nanostructure. Due to edge depletion from the reactive ion etch the width of the embedded 2DEG is reduced to about $w_{\text {eff }}=800 \mathrm{~nm}$. Beneath the points where the suspended beam is clamped, the supporting remainders of the sacrificial layer can also be discerned. The mesa is crossed by three equally suspended 75 nm wide Au electrodes (gate Nos. 1, 3, and 5) which can be used to deplete the 2DEG and form QPCs or tunneling barriers. The spacing between two adjacent crossing gates is 520 $\mathrm{nm}$. In addition, two suspended side gates (gate Nos. 2 and 4) of the same width are situated in between the crossing electrodes, enabling further control of the electron system.

The measurements detailed in the following illustrate the flexibility of the devices described and allow one to study the dissipation mechanisms in the phonon cavity for continuously varying electronic dimensions. First, the 2DEG was characterized by magnetotransport measurements. Then, depletion of one of the crossing gates was used to form a QPC. Subsequently, two neighboring crossing gates were employed as tunneling barriers to define a quantum dot (denoted $A$ in Fig. 1) which was weakly coupled to the reser-

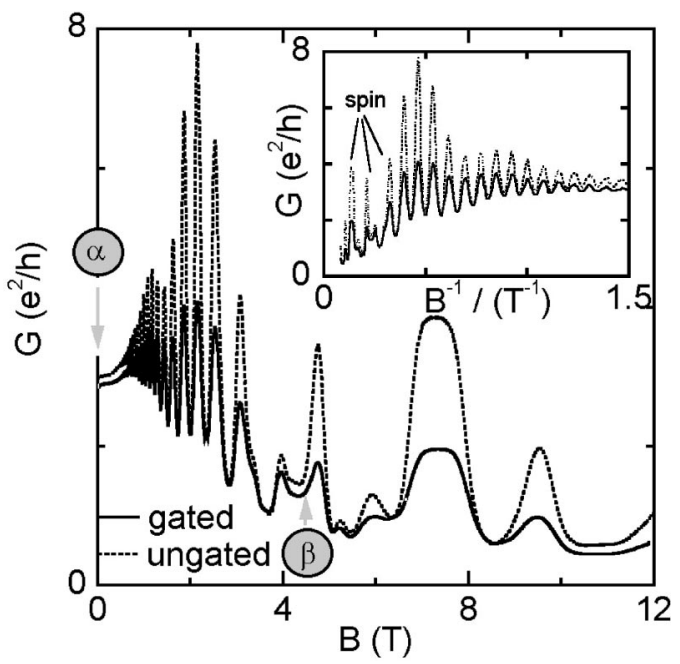

FIG. 2. Shubnikov-de Haas oscillations in conductance $G$ of a gated (solid line) and ungated but otherwise identical (dotted line) suspended 2DEG measured at $1.5 \mathrm{~K}$. The inset displays the same curves in a $1 / B$ representation, clearly showing spin-split filling factors of 3,5 , and 7 .

voirs, the energy levels of which could be tuned with one of the side gates. Beyond the single dot, all gate electrodes can be biased to define a fully tunable serial double $\operatorname{dot}(A$ and $B$, see Fig. 1).

In Fig. 2, the differential conductance $G=d I / d V_{\mathrm{SD}}$ is plotted versus a perpendicular magnetic field $B$, clearly showing resolved Shubnikov-de Haas oscillations down to $0.6 \mathrm{~T}$ at a temperature of $1.5 \mathrm{~K}$. The solid line shows a trace of the magnetoconductance for a gated beam like the one displayed in Fig. 1 whereas the dotted line represents that taken for an identical but ungated reference beam. Strikingly, not only is the zero field conductance almost identical in both cases, but the positions of the magnetoconductance minima that represent integer filling factors are also reached at the same magnetic field values. Hence, both the carrier density $n_{s}=g e / h \times 1 / \Delta(1 / B)=6.25 \times 10^{11} \mathrm{~cm}^{-2}$ with spin degeneracy factor $g=1$ as well as the mobility of the samples $\mu=\left(G_{0} / n_{s} e\right) \times l / w_{\text {eff }}=5500 \mathrm{~cm}^{2} / \mathrm{V} \mathrm{s}$, with $G_{0}$ being the zero field conductance, remain unaffected by the unbiased Schottky gates, which is strong evidence of the sample quality obtained. The reduced mobility compared to the sheet value is a result of the reactive ion etching and is observed for suspended and nonsuspended samples. ${ }^{8}$ Spin splitting is resolved for $\nu=7,5$, and 3 as can be seen from the inset that displays the same data versus $1 / B$.

Application of a negative bias to one of the crossing gates (e.g., gate No. 1 in Fig. 1) leads to the depletion of the underlying 2DEG and the number of conductance channels is successively reduced within the constriction. Since the 2DEG is not homogeneously depleted below the gate a conducting one-dimensional (1D) channel remains populated before pinchoff and forms a QPC. The differential conductance $G$ of the sample at $5 \mathrm{~K}$ is plotted as a function of $V_{g 1}$ by the solid line in Fig. 3(a) and it shows smooth conductance quantization steps before the 2DEG is pinched off at $V_{g 1}$ $=-2.0 \mathrm{~V}$. In the derivative of the conductance curve $d G / d V_{g 1}$ added to Fig. 3(a) as a dashed line four conductance steps are clearly identified as minima.

Figure 3(b) shows a comparison of the conductance of 

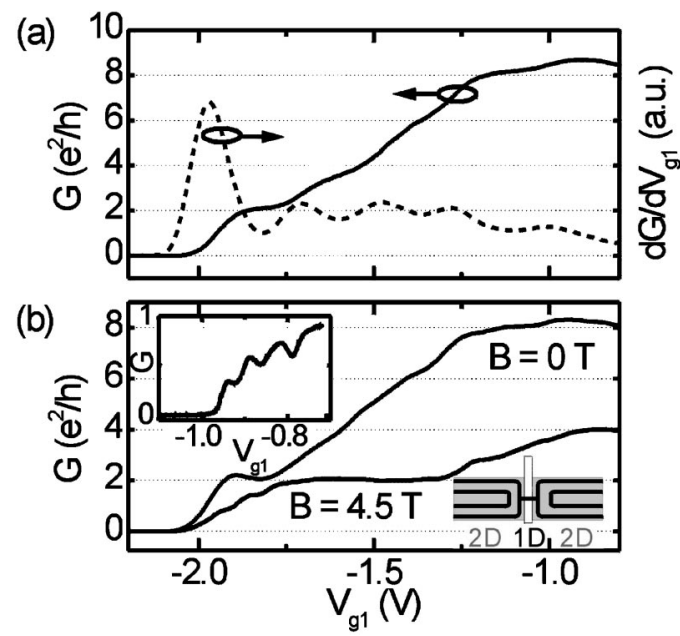

FIG. 3. (a) Conductance $G$ and derivative $d G / d V_{g 1}$ of the quantum point contact formed by depletion of gate No. 1 at $5 \mathrm{~K}$ showing four quantized conductance plateaus. (b) Conductance quantization at $1.5 \mathrm{~K}$ compared for $B=0 \mathrm{~T}$ and $4.5 \mathrm{~T}$ (positions $\alpha$ and $\beta$ in Fig. 2). The lower inset illustrates the interplay of Landau levels and 1D subbands. The upper inset displays Coulomb blockade oscillations with period of $2 E_{C} / \alpha$ (with conversion factor $\alpha=C_{g 1} / C_{\Sigma}$; see Ref. 8) in the quantum dot formed between gate Nos. 1 and 3 measured at $1.5 \mathrm{~K}$. The charging energy is $E_{C}=2.74 \mathrm{meV}$.

the QPC measured at $1.5 \mathrm{~K}$ for $B=0$ and $4.5 \mathrm{~T}$ which is equal to filling factor $\nu=7$ (marked as positions $\alpha$ and $\beta$ in Fig. 2, respectively). As expected quantization of magnetoelectric subbands results in stretching of the conductance plateaus. ${ }^{17}$ By performing this experiment edge channel transport and the transition through and dissipation in 1D constriction can be monitored [see the lower inset in Fig. $3(\mathrm{~b})]$.

As the next step, two of the electrodes (gates Nos. 1 and $3)$ were depleted enough to form tunneling barriers that enclose a quantum dot ( $A$ in Fig. 1) in the suspended beam. The upper inset in Fig. 3(b) displays Coulomb blockade oscillations in the conductance with respect to $V_{g 1}$ for fixed $V_{g 3}$ at $T=1.5 \mathrm{~K}$. Despite the strong background which is imposed on the oscillations the Coulomb diamond was mapped for varying $V_{g 1}$ and $V_{\mathrm{SD}}$. The charge energy of $E_{C}=e^{2} / 2 C_{\Sigma}=2.74 \mathrm{meV}$ deduced corresponds to a dot capacitance of $C_{\Sigma}=30 \mathrm{aF}$ and agrees well with the decomposition of the diamond above $6 \mathrm{~K}$.

In conclusion, we have demonstrated a fully gate-tunable low-dimensional electron system implemented into a freely suspended nanostructure. It was shown that the dimensionality of the system could be continuously reduced so that electron-phonon coupling of 2DEG, QPCs as well as quantum dots in a phonon cavity can be studied in a single device. In particular, tuning of the electron dwell time inside the quantum dot, which should strongly influence phonon emission, can be accomplished by variation of the tunnel coupling via the appropriate gating electrodes. Furthermore, the system can be expanded to a serial double dot structure similar to the unsuspended one discussed by Fujisawa et al. ${ }^{18}$ thereby allowing one to gain further insight into phononmediated dephasing processes.

Funds from the Bundesministerium für Forschung und Technologie (BMBF) under Contract No. 01BM914 are gratefully acknowledged.

${ }^{1}$ J. H. Davies, The Physics of Low-Dimensional Semiconductors (Cambridge University Press, Cambridge, 1998).

${ }^{2}$ A. N. Cleland, Foundations of Nanomechanics (Springer, Berlin, 2003).

${ }^{3}$ K. Schwab, E. A. Henriksen, J. M. Worlock, and M. L. Roukes, Nature (London) 404, 974 (2000).

${ }^{4}$ C. S. Yung, D. R. Schmidt, and A. N. Cleland, Appl. Phys. Lett. 81, 31 (2002).

${ }^{5}$ A. T. Tilke, L. Pescini, B. Lorenz, and R. H. Blick, Appl. Phys. Lett. (in press).

${ }^{6}$ S. Debald, T. Brandes, and B. Kramer, Phys. Rev. B 66, R041301 (2002).

${ }^{7}$ T. Vorrath, S. Debald, B. Kramer, and T. Brandes, cond-mat/0208493.

${ }^{8}$ J. Kirschbaum, E. M. Höhberger, R. H. Blick, W. Wegscheider, and M. Bichler, Appl. Phys. Lett. 81, 280 (2002).

${ }^{9}$ A. N. Cleland, J. S. Aldridge, D. C. Driscoll, and A. C. Gossard, Appl. Phys. Lett. 81, 1699 (2002).

${ }^{10}$ R. Knobel and A. N. Cleland, Appl. Phys. Lett. 81, 2258 (2002).

${ }^{11}$ R. G. Beck, M. A. Eriksson, M. A. Topinka, and R. M. Westervelt, Appl. Phys. Lett. 73, 1149 (1998).

${ }^{12}$ J. G. E. Harris, R. Knobel, K. D. Maranowski, A. C. Gossard, N. Samarth, and D. D. Awschalom, Phys. Rev. Lett. 86, 4644 (2001).

${ }^{13}$ H. X. Tang, X. M. H. Huang, and M. L. Roukes, Appl. Phys. Lett. 81, 3879 (2002).

${ }^{14}$ E. M. Höhberger, R. H. Blick, F. W. Beil, W. Wegscheider, M. Bichler, and J. P. Kotthaus, Physica E 12, 487 (2002).

${ }^{15}$ R. H. Blick, F. G. Monzon, W. Wegscheider, M. Bichler, F. Stern, and M. L. Roukes, Phys. Rev. B 62, 17103 (2000).

${ }^{16}$ E. M. Höhberger, J. Kirschbaum, R. H. Blick, J. P. Kotthaus, and W. Wegscheider, Physica E 18, 99 (2003).

${ }^{17}$ B. J. van Wees, L. P. Kouwenhoven, H. van Houten, C. W. J. Beenakker, J. E. Mooij, C. T. Foxon, and J. J. Harris, Phys. Rev. B 38, 3625 (1988).

${ }^{18}$ T. Fujisawa, T. H. Oosterkamp, W. G. van der Wiel, B. W. Broer, R. Aguado, S. Tarucha, and L. P. Kouwenhoven, Science (Washington, DC, U.S.) 282, 932 (1998). 